\title{
Producción del Ensayo Argumentativo en Estudiantes de dos Establecimientos de Educación Media Técnico-Profesional de la Provincia de Núble. Resultados de un Diagnóstico*
}

\author{
Federico Carlos Pastene Labrín** \\ Rosa Marta Díaz Chavarría*** \\ Sandra Annette Molina Castillo****
}

\section{Resumen}

El artículo presenta los resultados de una evaluación aplicada a estudiantes de $3^{\circ}$ y $4^{\circ}$ año de dos establecimientos de Educación Media Técnico-Profesional (EMTP) de la Provincia de Nuble, con la finalidad de determinar el dominio en la producción escrita de un ensayo argumentativo. Se realizó una investigación cuantitativa descriptiva con un diseño transversal a una población compuesta por 202 alumnos. Los resultados demuestran un desempeño aceptable en la producción de un ensayo con un $60 \%$ de logro en la muestra total, donde la dimensión mejor lograda es adecuación y las más descendidas son partes del discurso y argumentación, revelando dificultades para escribir y comunicar ideas, reconocer y aplicar la estructura básica del discurso argumentativo junto con el desarrollo del pensamiento analítico y reflexivo.

Palabras clave: Evaluación, producción de textos, ensayo argumentativo, educación técnico-profesional.

\section{Argumentative Essay Production in Students from two Technical Professional High Schools of Ñuble Province. Results of a Diagnosis}

\begin{abstract}
The article presents the results of evaluation applied to 3rd and 4th graders of two technical professional high schools of Nuble province, with the aim to determine the domain in written production of an argumentative essay. A descriptive quantitative research was held
\end{abstract}

* Este artículo proviene de una investigación patrocinada por el Convenio de Desempeño: “Sistema Territorial de Educación: Una plataforma de articulación prospectiva para la competitividad y el desarrollo equitativo y sustentable de la región del Biobío". Proyecto UBB 1202, de la Universidad del Bío-Bío-MINEDUC. Se agradece la asesoría estadística del Dr. Daniel Tello.

** Chileno. Doctor en Ciencias Humanas Mención Discurso y Cultura de la Universidad Austral de Chile. Académico Universidad del Bío-Bío, Chillán, Chile. fpastene@ubiobio.cl

*** Chilena. Magíster en Literatura de la Universidad de Chile. Académica Universidad del Bío-Bío, Chillán, Chile.rdiaz@ubiobio.cl

**** Chilena. Magíster en Educación. Universidad La República, Chile. Académica Universidad del BíoBío, Chillán, Chile.smolina@ubiobio.cl 
with a cross design to a group composed of 202 students. The results show an acceptable performance in an essay production with a $60 \%$ of the total sample, where the best achieved dimension is adaptation and the lowest ones parts of speech and argument, revealing difficulties to write and communicate ideas, recognize and apply basic structures of argumentative discourse together with the development of analytic and reflexive thinking.

Keywords: Evaluation, text production, argumentative essay, technical professional education.

Recibido: 01-02-2016

Aceptado: 16-06-2016 


\section{Introducción}

Desde la Reforma educacional de los '90 en Chile, en la Enseñanza Media operó un cambio de paradigma en relación con el área de lenguaje y comunicación, al enfatizar el aprendizaje y desarrollo de las competencias comunicativas desde el enfoque comunicativo-funcional por sobre la enseñanza de contenidos lingüísticos y literarios desde una perspectiva gramatical, histórica y filológica, respectivamente. Tanto el Marco Curricular (2009) como las recientes Bases Curriculares (MINEDUC, 2013a) hacen hincapié en que durante la trayectoria educativa desempeñan un papel fundamental el aprendizaje de la lengua y el desarrollo de las competencias comunicativas, culturales y de reflexión sobre el lenguaje y los textos, necesarios para una vida plena y una participación libre, crítica e informada en la sociedad contemporánea. Una de estas habilidades comunicativas centrales en la formación de los estudiantes es la escritura, principal forma de transmitir y preservar el conocimiento que, además de satisfacer otras importantes necesidades como expresar la interioridad y desarrollar la creatividad, abre la posibilidad de comunicarse sin importar el tiempo y la distancia. En otras palabras, es un instrumento eficaz para convencer a otros, entre otras funciones (Bases Curriculares, MINEDUC, 2013a).

Por su parte, en la Educación Media, junto con la Educación Científico-Humanista, está incluido un ámbito de Formación Diferenciada, que corresponde a canales de especialización enfocados en las necesidades y áreas de interés de cada estudiante. Esta es la Formación Técnico-Profesional, que ofrece a los alumnos y las alumnas oportunidades de realizar aprendizajes en un campo de especialización que facilite su acceso a un primer trabajo remunerado, atendiendo a sus intereses, aptitudes y disposiciones vocacionales, mediante una formación técnica en el ámbito de un sector del mundo productivo, pero que, por cierto, no se abandonan ciertas competencias básicas indispensables para su desarrollo como la de "Comunicarse oralmente y por escrito con claridad, utilizando registros de habla y escritura pertinentes a la situación laboral y a la relación con los interlocutores" (MINEDUC, 2013b., p. 13).

Sin embargo, y aunque se declara la relevancia formativa de la escritura en los instrumentos curriculares del Ministerio de Educación, 
no existe suficiente evidencia del desempeño de esta competencia en los estudiantes en el sistema escolar, sobre todo de tercer y cuarto año medio, que pronto se proyectan, la mayoría de ellos, hacia la prosecución de estudios superiores, ya sea universitarios, técnicos o de otra área como el ingreso a las fuerzas armadas, donde esta habilidad es fundamental. Este vacío se ve incrementado por el énfasis que existe actualmente en la medición de la lectura por el SIMCE -excepto en $6^{\circ}$ Básico-, y la PSU, que presionan a reforzar la comprensión lectora en el aula por sobre las otras habilidades.

En el ámbito de la educación media técnico-profesional de nuestro país (EMTP), es evidente el rezago que ha tenido desde las políticas públicas este relevante sector educacional (CEPPE, 2013). Weinstein (2013) plantea que esta opción educativa es elegida por cerca del 45\% de los jóvenes que asisten a tercero y cuarto medio, y la gran mayoría de ellos -el 91\% - proviene de los dos quintiles de ingresos más pobres de la población. El autor agrega que las condiciones de aguda vulnerabilidad social están estrechamente correlacionadas con los bajos rendimientos escolares, reflejados en que el promedio nacional SIMCE de $2^{\circ}$ medio de la EMTP de 2012, 2010, 2008 y 2006 es 25 puntos inferior al que obtienen los jóvenes que ingresan a la Enseñanza Media Científico Humanista (EMCH). En consecuencia, la EMTP está convocada a entregar una formación integral que permita a los jóvenes no solo insertarse en el mercado laboral, sino también formarse como personas y como ciudadanos, y contar con las herramientas necesarias para proseguir estudios superiores, donde el dominio de las competencias comunicativas juegan un rol clave. Más aún, adquiere relevancia el poder indagar y generar evidencias acerca de esta macrohabilidad en el contexto escolar.

En este sentido, el presente artículo tiene como objetivo central exponer los resultados de una investigación que determinó el nivel de desempeño en la producción de un ensayo argumentativo en estudiantes de $3^{\circ}$ y $4^{\circ}$ año de dos establecimientos de Educación Media TécnicoProfesional de la Provincia de Nuble. Asimismo, se pretende abordar críticamente los resultados obtenidos y efectuar propuestas de abordaje futuro vinculadas con la competencia escrita. 


\section{Antecedentes}

\subsection{Evaluación de la escritura en el sistema educativo reciente. Algunos datos}

El Ministerio de Educación ha generado mecanismos para medir la calidad de la educación y así contribuir al fortalecimiento educativo. Un primer instrumento es el SIMCE, que desde 1988 provee de información relevante para su quehacer a los distintos actores del sistema educativo, informando sobre los logros de aprendizaje de los estudiantes en diferentes áreas de aprendizaje del currículo nacional, y relacionándolos con el contexto escolar y social en el que estos aprenden. En el área de lenguaje y comunicación, ha sido relevante la evaluación de la lectura -mayoritariamente-, y solo desde 2012 se mide la escritura de manera estable e independiente en $6^{\circ}$ Básico. Los resultados del SIMCE de esta habilidad en 2014 arrojaron los siguientes resultados: cuando se mide el desarrollo de ideas y vocabulario, solo el $12 \%$ alcanza el nivel 4 , que es el más alto, mientras que el 39\% llega al nivel 3, el 30\% al nivel 2, y el $7 \%$ al nivel más bajo, con una omisión de respuestas del 12\%. En tanto, cuando se mide cohesión, es decir, la capacidad de usar conectores de manera óptima, solo el 14\% de los niños alcanza el nivel 4, que es el más alto, mientras el 35\% llega al nivel 3 y el 32\% al nivel 2. En cuanto a las fortalezas, un $51 \%$ de los estudiantes obtuvo el nivel 4 en adecuación del propósito comunicativo. En total, los estudiantes obtuvieron un puntaje de 50 puntos que, asimilados al Simce tradicional, equivale a 250 puntos (cfr. Agencia, 2015). Esto refleja, sin duda, que aún falta en la educación básica fortalecer esta importante habilidad transversal y generar mediciones en los cursos superiores.

Desde el ámbito latinoamericano, destaca la evaluación realizada por LLECE (Laboratorio Latinoamericano de Evaluación de la Calidad de la Educación) que publicó en julio de 2015 el Tercer Estudio Regional Comparativo y Explicativo (TERCE), donde unas delas habilidades medidas fue la escritura en tercer grado de primaria en 15 países (las otras fueron lectura, matemática y ciencias). Chile logró un puntaje promedio de 3,23 de 4, ubicándose por sobre la media de las naciones evaluadas que presentan un puntaje de 2,86. En relación con las puntuaciones promedio y comparación de las puntuaciones de cada país en los tres dominios de la prueba de escritura, Chile obtuvo un 3,18 en dominio discursivo, 3,31 
dominio textual y 3,18 convenciones de legibilidad, sobrepasando de todos modos el promedio de los otros países en estos tres dominios. Estos resultados tienden a favorecer al país en comparación con sus símiles, pero aún no se consideran los otros niveles educativos que esperan evidenciar un registro de sus avances.

En la Enseñanza Media, el SIMCE mide lectura solo en $2^{\circ}$ Medio, lo que ha derivado en que se tome a los resultados de la PSU como indicadores de la calidad educativa, conduciendo a los establecimientos educacionales ser rankeados muchas veces con fines promocionales, información que patentiza la brecha que existe entre los colegios municipales y particulares pagados, aun cuando es una prueba de selección para el ingreso a la universidad. Esta Prueba de Selección Universitaria (PSU) considera dos ítemes con los cuales se pretende medir la producción de textos: conectores y plan de redacción con 10 y 15 preguntas respectivamente, de un total de 80 , lo que representa un $31,25 \%$ del total del instrumento. Sin embargo, no cuenta con la redacción de un texto propiamente tal. Hace algunos años que se viene anunciando la posibilidad de incluir una sección de producción textual. En 2006 y 2007 se realizaron, por parte del DEMRE, unas investigaciones exploratorias sobre el tema, cuyas conclusiones indican que se requiere de decisiones operativas como el mayor tiempo de corrección y el diseño de un instrumento que sirva como complementario a la PSU (Hernández, 2009). En 2012 se anunciaban profundos cambios en la PSU, como la inclusión de un ensayo escrito (La Segunda 4.1.2012) que al transcurrir el tiempo se ha diluido por razones logísticas: difícil de revisar escritos de un volumen de alumnos que bordean los 250.000 (DEMRE, Intersección curricular..., 2014). Por lo tanto, desde este parámetro han habido estudios e intentos por incluir la escritura como una dimensión que debe considerarse al ingreso de los estudios universitarios.

En suma, se constata que en nuestro país los resultados que arroja la medición de la escritura indican que aún se debe afianzar pedagógicamente esta competencia, cuya evidencia principalmente es visible en la Educación Básica y no tanto en la Educación Media; para esta última, se toman como parámetros mediciones efectuadas por las instituciones superiores a sus alumnos novatos, pero se reitera la inexistencia de pesquisas de la comunicación escrita a nivel del sistema escolar, sobre todo en los últimos años de la Educación Media. 


\subsection{La producción escrita}

Para explicar en qué consiste la habilidad comunicativa de escribir, han surgido diferentes enfoques y modelos que, desde una dimensión de la producción del texto como producto o como proceso, orientan, por un lado, la descripción de la naturaleza de la escritura y, por otro, constituyen referentes empleados en el abordaje pedagógico (Parodi, 2003). En el sistema escolar se concibe, a grandes rasgos, a la escritura no como una mera destreza lingüística, sino como un proceso que involucra la producción de diversos tipos de textos (o géneros discursivos), teniendo en consideración un propósito y la situación/contexto comunicativo, la recepción del texto y su destinatario, relevando el fortalecimiento de la habilidad del estudiante en ser capaz de expresar sus ideas y pensamientos de modo coherente y con respeto a las convenciones de la lengua. Sin duda, esta orientación actual del fenómeno es producto del enfoque comunicativo funcional que prima en el currículum vigente. Pero escribir no es una competencia sencilla que se logra de un momento a otro. Involucra una serie de demandas cognitivas y factores asociados.

Para Kellog \& Whiteford (2012), el sujeto que está en franco avance en la tarea de escribir debe ser capaz de planificar ideas, generar textos y revisarlos, siendo un proceso recursivo. Además, esta habilidad involucra activar en la memoria de trabajo una representación mental de la macroestructura semántica en contacto con la memoria de largo plazo, con el 'almacén cognitivo' que surtirá de elementos para su tarea, sin dejar de lado la atención. La representación acerca del autor, texto y lector se activan y actualizan a medida que se avanza en la redacción. Por su parte, en cuanto a los factores relevantes del proceso, se deben tener en cuenta los contextos y los géneros discursivos, el conocimiento acerca del tema y de la tarea de escritura, como además la organización y elaboración de las ideas, sumado a los aspectos afectivos como la actitud hacia el tema y la motivación; es decir, la puesta en funcionamiento de estrategias cognitivas, metacognitivas y afectivas de producción textual.

En la concreción de estos postulados, por ejemplo, en un instrumento curricular, como es el texto del estudiante de lenguaje y comunicación, se observa una serie de actividades que comprometen el desarrollo de la escritura considerando los recursos antes expuestos (Cfr. Cofré et al., 2013). Sin embargo, la superación del enfoque como producto de la escritura ha sido un proceso lento dentro de las aulas chilenas, que 
gracias a estos recursos pedagógicos se ha podido avanzar. Un primer aporte para cambiar este paradigma al de la escritura como proceso, ha sido, sin duda, el abordaje didáctico del modelo cognitivo de Flower y Hayes (1981), que tiene como puntos convergentes el de considerar al escritor y sus procesos mentales en un proceso recursivo y no lineal. Además, requiere una autorregulación (metaproducción) por parte del escritor en cuanto a sus conocimientos, el tema, el vocabulario, el texto, el destinatario, el propósito comunicativo, junto con las etapas de planificación, traducción y revisión.

Pero este modelo ha recibido críticas, ya que no profundiza en la dimensión sociocultural que el proceso de escribir también implica (Errázuriz, 2014). Es Grabey Kaplan (1996) quienes logran una articulación en tres ámbitos al postular un modelo comunicativo del proceso de escritura, con constituyentes cognitivos, sociales y textuales. En otras palabras, es necesario integrar las tres mayores consideraciones para una teoría de la escritura, las que son: el procesamiento cognitivo del escritor, los recursos lingüísticos y textuales que se activan en la composición y los factores de carácter contextual que precisan las particularidades de la escritura (Cfr. Neira, 2011).

En suma, la escritura involucra la inclusión de aspectos cognitivos, textuales y contextuales, donde la meta de producir un texto requiere de un sujeto que planifica, organiza y revisa constantemente hasta obtener un producto convincente con recursos que la lengua le brinda. El escritor es capaz de monitorear su proceso, que es indudablemente recursivo, pero que, en el caso de su aprendizaje en el aula, requiere de modelos didácticos que brinden un andamiaje necesario en el fortalecimiento de esta competencia en los estudiantes.

\subsection{El ensayo argumentativo}

El ensayo es un género discursivo que ha sido abordado a través de múltiples perspectivas -ya sea desde la literatura, pasando por la filosofía y las ciencias-; por lo tanto, se puede decir que no existe el ensayo, sino diferentes tipos de ensayos. Desde que fue creado por Michel de Montaigne (1533-1592) y plasmado a través de sus famosos Essais (1580), el texto ensayístico ha ido, a través de tiempo, adquiriendo las fisonomías que le han la otorgado las épocas, las corrientes estéticas y las disciplinas. Para la RAE (2014) es un "Escrito en prosa en el cual un autor desarrolla 
sus ideas sobre un tema determinado con carácter y estilo personales". Por su parte, Weinberg (2006, p. 20) lo concibe como: "texto en prosa que manifiesta un punto de vista bien fundamentado, bien escrito y responsable del autor respecto de algún asunto del mundo". En otras palabras, este corresponde a un tipo de texto en el cual el escritor expresa sus propias reflexiones frente a determinados temas con un estilo propio; es decir, dirige el sentido de la prosa según sus preferencias.

En la Educación Media, los estudiantes tienen un acercamiento al ensayo por lo menos desde tres vías. En primer lugar, cuando en alguna asignatura -ya sea de lenguaje, historia, ciencias o de otra área curricular- los alumnos deben redactar un ensayo como una actividad o instrumento de evaluación donde sean capaces de evidenciar determinados aprendizajes. En segundo lugar, cuando se trabaja el discurso argumentativo, por ejemplo, al leer, analizar y producir el ensayo como un texto secuencia textual argumentativa (tesis, argumentos, conclusión) (Cfr. Guin-Po et al., 2011). Y, en tercer lugar, la lectura de ensayos como parte del canon literario, donde se valora su conformación estética y cultural, para luego producir estos textos con intención literaria (Cfr. Domínguez, 2013 et al.). De estos horizontes, se rescata el sentido del ensayo como texto argumentativo, ya que, siguiendo la estrategia estructural para producir textos, brinda las claves necesarias para que este tipo de texto sea elaborado. Por lo tanto, es un texto que se aborda pedagógicamente en el aula, según las orientaciones curriculares y los énfasis que el docente opte otorgarles.

Para esta investigación, se empleó la configuración textual y genérica que han trabajado Flotts y Manzi (2012), Preiss et al. (2013) y Errázuriz (2014), con un esquema argumentativo global (o modelo genérico del ensayo argumentativo) que contiene los elementos básicos de la argumentación, el ensayo y la escritura del mismo: tesis, cuerpo argumentativo y una conclusión (Ducrot, 2001; Lo Cascio, 1998; Van Dijk, 1997). Siguiendo a Errázuriz (2014), se concibe en el despliegue de la estructura del texto ensayístico, el siguiente orden:

En primer lugar, un marco inicial -que corresponde a la introducción y exhibe la controversia- puede estar conformado por elementos que son opcionales, como la presentación del tema, los objetivos o la descripción del problema. En segundo lugar, el desarrollo del tema, 
el cual corresponde al cuerpo de la argumentación y muestra las posiciones a favor o en contra, puede estar integrado por uno o más argumentos conectados con uno o más contraargumentos. Por último, el marco final -que corresponde a la conclusión y clausura la argumentaciónpuede encontrarse componentes que son opcionales, como el cierre del tema, la síntesis, la discusión, las proyecciones y la recapitulación. En cualquiera de estas etapas se puede incluir la tesis. (p. 220-221).

Por lo tanto, este modelo textual de ensayo argumentativo es el que se consideró en esta investigación, puesto que su estructura básica debe ser conocida por los estudiantes. Asimismo, este esquema ha sido ampliamente aplicado, validado y estudiado, constituyéndose en un modelo orientador que permite adaptarlo según las necesidades y los contextos de nuevas investigaciones en escritura, (en este caso, de escritura escolar).

\section{Diseño metodológico}

Este estudio tuvo como objetivo principal determinar el nivel de dominio de producción textual de estudiantes de $3^{\circ}$ y $4^{\circ}$ año de dos establecimientos de Educación Media Técnico Profesional de la Provincia de Nuble, mediante la aplicación de una prueba diagnóstica de escritura donde se debió redactar un ensayo argumentativo, proceso efectuado durante el año 2015. Por consiguiente, es una investigación con un enfoque cuantitativo, de alcance principalmente descriptivo y con un diseño no experimental transversal (Hernández et al., 2006). Se recurre a métodos estadísticos descriptivos, inferenciales y correlacionales mediante el empleo de Excel y el Programa R (R Core Team, 2013; Fox, J., 2005).

\subsection{Población o sujetos de estudio}

La población estuvo compuesta por 202 estudiantes de $3^{\circ}$ y $4^{\circ}$ año de dos establecimientos de Educación Media Técnico-Profesional (EMTP) de la Provincia de Nuble. El Establecimiento 1 es de dependencia delegada que se encuentra en la ciudad de Chillán, donde se consideraron dos terceros medios y dos cuartos medios con las especialidades de Administración, Contabilidad, Secretariado y 
Ventas, conformando un total de 104 estudiantes, con un promedio de 26 alumnos por curso, que representa un 51,4\% del total de la población evaluada en la investigación. Asimismo, $31(29,8 \%)$ son hombres y 85 $(70,2 \%)$ mujeres, con un promedio de Notas de Enseñanza Media (NEM) 5,3 y notas promedio de lenguaje en la Enseñanza Media de 5,2. Por su parte, el Establecimiento 2, de dependencia municipal, se encuentra en otra comuna de la Provincia de Nuble, y también se consideraron dos terceros medios y dos cuartos medios con las especialidades de Elaboración Industrial de Alimentos y Servicio de Alimentación Colectiva, contándose un total de 98 estudiantes, con un promedio de 24 alumnos por curso, lo que corresponde al $48,6 \%$ del total medido. 28 son hombres $(28,5 \%)$ y $70(71,5 \%)$ mujeres, con un NEM de 5,2 y notas de lenguaje de Enseñanza Media de 4,9. Aunque las cifras parecieran ser similares en algunos puntos, destaca el hecho de que en ambos establecimientos la población abordada sean más mujeres que hombres, correspondiendo al $70,8 \%$ y al $29,2 \%$, respectivamente.

\subsection{Instrumento}

Para evaluar el desempeño en comunicación escrita, se elaboró y aplicó una prueba de escritura, de papel y lápiz, con una instrucción que incluyó la propuesta de cuatro temas, donde el estudiante debió elegir uno de ellos para redactar -en dos planas, al menos- su postura. Para su revisión se diseñó una rúbrica con las dimensiones adaptadas del instrumento que aplica MIDE UC (Flotts y Manzi, 2012; Preiss et al., 2013; Errázuriz, 2014), a saber, ortografía, coherencia, cohesión, adecuación, léxico, partes del discurso, argumentación y cobertura. Además, se estructuró en una escala de 1 a 5, correspondiendo a cada cifra un nivel de desempeño respectivamente: $1=$ Deficiente; $2=$ Limitado; 3=Aceptable; $4=$ Adecuado y 5=Destacado, adaptada también de MIDE UC. Para la categorización global de los resultados de la muestra, se tomaron en cuenta los mismos niveles y se diseñó una rúbrica holística (Mertler, 2001). Los insumos generados para evaluar los ensayos fueron debidamente validados mediante la revisión por parte de expertos y la aplicación piloto a una muestra, cuya confiabilidad resultó ser elevada (Alfa de Cronbach $\alpha=0,86$ ). 


\section{Resultados}

\subsection{Resultados de porcentaje de logro y por niveles de desempeño}

Los porcentajes de logro promedio en las ocho dimensiones medidas en la prueba de escritura de un ensayo argumentativo se presentan en la Tabla 1. Sobre la base de estos datos es posible presentar cuáles fueron aquellos de mayor y menor logro, así como comparar los resultados de los estudiantes según establecimiento educacional.

Tabla 1.

Porcentajes de logro en las dimensiones evaluadas según establecimiento educacional, cursos y especialidades.

\begin{tabular}{lccccccccccc}
\hline & \multicolumn{3}{c}{ Establecimiento $1(\mathrm{n}=104)$} & \multicolumn{7}{c}{ Establecimiento $2(\mathrm{n}=98)$} \\
\hline & \multicolumn{3}{c}{ Curso y Especialidad } & \multicolumn{7}{c}{ Curso y Especialidad } \\
\hline & $3^{\circ}$ & $3^{\circ}$ & $4^{\circ}$ & $4^{\circ}$ & & $3^{\circ}$ & $3^{\circ}$ & $4^{\circ}$ & $4^{\circ}$ & \\
\hline Dimensión & ADM & CON & SEC & VEN & M & EIA & SAC & EIA & SAC & M & TOT \\
\hline Ortografía & 68,5 & 71,7 & 63,0 & 63,7 & $\mathbf{6 6 , 7}$ & 58,4 & 59,3 & 59,1 & 54,8 & $\mathbf{5 7 , 9}$ & $\mathbf{6 2 , 3}$ \\
\hline Coherencia & 62,3 & 79,2 & 66,7 & 65,9 & $\mathbf{6 8 , 5}$ & 44,0 & 56,3 & 67,8 & 55,7 & $\mathbf{5 6 , 0}$ & $\mathbf{6 2 , 2}$ \\
\hline Cohesión & 56,9 & 69,2 & 61,5 & 53,3 & $\mathbf{6 0 , 2}$ & 40,0 & 58,5 & 67,8 & 54,8 & $\mathbf{5 5 , 3}$ & $\mathbf{5 7 , 8}$ \\
\hline Adecuación & 60,0 & 78,3 & 68,9 & 72,6 & $\mathbf{7 0 , 0}$ & 51,2 & 68,9 & 81,7 & 59,1 & $\mathbf{6 5 , 2}$ & $\mathbf{6 7 , 6}$ \\
\hline Léxico & 54,6 & 65,8 & 64,4 & 62,2 & $\mathbf{6 1 , 8}$ & 46,4 & 60,7 & 71,3 & 51,3 & $\mathbf{5 7 , 4}$ & $\mathbf{5 9 , 6}$ \\
\hline $\begin{array}{l}\text { Partes del } \\
\text { Discurso }\end{array}$ & 56,2 & 45,8 & 61,5 & 51,1 & $\mathbf{5 3 , 7}$ & 44,0 & 63,0 & 68,7 & 51,3 & $\mathbf{5 6 , 8}$ & $\mathbf{5 5 , 2}$ \\
\hline Argumentación & 58,5 & 54,2 & 60,7 & 62,2 & $\mathbf{5 8 , 9}$ & 43,2 & 56,3 & 64,3 & 47,0 & $\mathbf{5 2 , 7}$ & $\mathbf{5 5 , 8}$ \\
\hline Cobertura & 57,7 & 54,2 & 70,4 & 49,6 & $\mathbf{5 8 , 0}$ & 72,2 & 68,1 & 75,7 & 62,6 & $\mathbf{6 9 , 7}$ & $\mathbf{6 3 , 8}$ \\
\hline $\begin{array}{l}\text { Promedio } \\
\text { Producción }\end{array}$ & & & & & $\mathbf{6 2 , 2}$ & & & & & $\mathbf{5 8 , 9}$ & $\mathbf{6 0 , 5}$ \\
\hline
\end{tabular}

Nota: Las especialidades son Administración (ADM), Contabilidad (CON), Secretariado (SEC), Ventas (VEN), Elaboración Industrial de Alimentos (EIA) y Servicio de Alimentación Colectiva (SAC). 
En términos porcentuales totales, la mayor dimensión lograda es Adecuación, con un 67,6\%, y el logro más descendido es Partes del Discurso, con un 55,2\%, lo que significa que la gran mayoría concretó el propósito comunicativo previsto, pero no así dividió el texto en la tríada de introducción, desarrollo y conclusión, secuencia presente en el modelo genérico de ensayo utilizado. Por su parte, la mejor dimensión lograda en el Establecimiento 1 es Adecuación 70\%, y la más baja es Partes del Discurso con 53,7\%. Esto significa que un número significativo de estudiantes cumplió con el propósito comunicativo del texto, no así con la división del escrito en tres momentos. En cambio, en el Establecimiento 2, la dimensión más lograda es Cobertura, con un $69,7 \%$, y la más descendida es Argumentación, con un 52,7\%, es decir, un poco más de la mitad de los evaluados logró considerar los recursos para desarrollar un buen texto argumentativo. En suma, se obtiene un $60,5 \%$ de dominio en la competencia de escribir un ensayo argumentativo por los dos establecimientos considerados, donde el $\mathrm{N}^{\circ} 1$ obtiene el mayor logro, con un $62,2 \%$, y el $\mathrm{N}^{\circ} 2$ solo con un $58,9 \%$. Esto refleja, desde el punto de vista territorial, que la unidad educativa que se encuentra en la ciudad de Chillán, capital de la Provincia de Ñuble, tiene mejor desempeño que aquel establecimiento que pertenece a otra comuna de la Provincia.

Por otra parte, los resultados que se obtienen de la investigación realizada pueden ser descritos a partir de la presentación de los puntajes asignados por nivel de desempeño en comunicación escrita (1 a 5) en la Tabla 2, considerando las ocho dimensiones medidas en la prueba de escritura de un ensayo argumentativo y también los puntajes globales. En efecto, esto permite conocer los distintos niveles de desempeño, así como comparar los resultados de los estudiantes con su correspondiente curso y especialidad. 
Tabla 2.

Puntajes de desempeño obtenidos en las dimensiones evaluadas según establecimiento educacional, cursos y especialidades.

\begin{tabular}{|c|c|c|c|c|c|c|c|c|c|c|c|c|}
\hline & \multicolumn{5}{|c|}{ Establecimiento $1(\mathrm{n}=104)$} & \multicolumn{5}{|c|}{ Establecimiento $2(\mathrm{n}=98)$} & & \\
\hline & \multicolumn{4}{|c|}{ Curso y Especialidad } & \multicolumn{6}{|c|}{ Curso y Especialidad } & & \\
\hline & $3^{\circ}$ & $3^{\circ}$ & $4^{\circ}$ & $4^{\circ}$ & & $3^{\circ}$ & $3^{\circ}$ & $4^{\circ}$ & $4^{\circ}$ & & & \\
\hline Dimensión & $\mathrm{ADM}$ & $\mathrm{CON}$ & SEC & VEN & $\mathbf{M}$ & EIA & SAC & EIA & SAC & $\mathbf{M}$ & TOT & D.S. \\
\hline Ortografía & 3,7 & 3,6 & 3,1 & 3,2 & 3,4 & 2,9 & 3,0 & 3,0 & 3,0 & 3,0 & 3,2 & 1,26 \\
\hline Coherencia & 3,4 & 4,0 & 3,3 & 3,3 & 3,5 & 2,2 & 2,8 & 3,4 & 3,0 & 2,9 & 3,2 & 1,04 \\
\hline Cohesión & 3,1 & 3,5 & 3,1 & 2,7 & 3,1 & 2,0 & 2,9 & 3,4 & 3,0 & 2,8 & 3,0 & 0,94 \\
\hline Adecuación & 3,3 & 3,9 & 3,4 & 3,6 & 3,6 & 2,6 & 3,4 & 4,1 & 3,2 & 3,3 & 3,5 & 1,02 \\
\hline Léxico & 3,0 & 3,3 & 3,2 & 3,1 & 3,2 & 2,3 & 3,0 & 3,6 & 2,8 & 2,9 & 3,1 & 0,89 \\
\hline $\begin{array}{l}\text { Partes del } \\
\text { Discurso }\end{array}$ & 3,0 & 2,3 & 3,1 & 2,6 & 2,8 & 2,2 & 3,1 & 3,4 & 2,8 & 2,9 & 2,8 & 1,29 \\
\hline Argumentación & 3,2 & 2,7 & 3,0 & 3,1 & 3,0 & 2,2 & 2,8 & 3,2 & 2,6 & 2,7 & 2,9 & 1,09 \\
\hline Cobertura & 3,1 & 2,7 & 3,5 & 2,5 & 3,0 & 3,6 & 3,4 & 3,8 & 3,4 & 3,6 & 3,2 & 1,17 \\
\hline $\begin{array}{l}\text { Promedio } \\
\text { Producción }\end{array}$ & 3,2 & 3,2 & 3,2 & 3,0 & 3,2 & 2,5 & 3,1 & 3,5 & 3,0 & 3,0 & 3,1 & 1,26 \\
\hline
\end{tabular}

En términos generales, los dos establecimientos obtuvieron un promedio total de 3,1 puntos, que corresponde a un desempeño aceptable; es decir, los estudiantes son capaces de producir un ensayo argumentativo considerando de modo básico la mayoría de las dimensiones o criterios evaluados. Aunque emplean las convenciones ortográficas, brindan al texto coherencia y cohesión para que sea comprensible, a la vez que dan cuenta del propósito comunicativo del texto, con un léxico suficiente y un espacio razonable, siendo limitado el manejo de las formas básicas que estructuran la entidad discursiva ensayo como texto y argumento ( 2,8 en Partes del Discurso y 2,9 en Argumentación).

En el establecimiento 1, solo Partes del Discurso, con un 2,8, está catalogada como desempeño limitado. Todas las demás se reúnen en la categoría de desempeño aceptable. No así el establecimiento 2, que en cinco dimensiones (Coherencia, Cohesión, Léxico, Partes del Discurso y Argumentación) tienen un desempeño limitado -más que la unidad 
educativa anterior-, aunque en su promedio final no logran descender de un 3,0. Con respecto a los cursos y especialidades, es el $3^{\circ}$ Ventas del Establecimiento 1 que logra la puntuación más alta en Coherencia, con un 4,0 (Adecuado), y la más baja en Partes del Discurso, con 2,3 (Limitado). En cambio, en el Establecimiento 2, el curso $3^{\circ}$ de la Especialidad Elaboración Industrial de Alimentos -en las siete primeras dimensiones- sus puntajes corresponden a desempeños limitados, y solo en Cobertura con un 3,6 es aceptable, frente al curso $4^{\circ}$ de la misma especialidad, que alcanza la puntuación más alta del colegio con 4,1 en Adecuación (desempeño adecuado). En suma, este último grupo es el que obtiene el mejor puntaje de la población evaluada con un 3,5 (desempeño aceptable).

Por último, en la variabilidad de los resultados, la dimensión que presenta un desempeño más homogéneo es Léxico con una D.E. $=0,89$, y el más heterogéneo es Partes del Discurso con una D.E.=1,29. Sin embargo, las desviaciones tienden a ser similares en ambos establecimientos educacionales.

\subsection{Relación de algunas variables con el desempeño obtenido en la producción de un ensayo argumentativo}

En la investigación realizada, también se encuestó y registró información de otras variables presentes en la población en estudio que, de igual modo, pueden ser analizadas desde los métodos estadísticos inferenciales. En relación con la influencia de factores individuales en la habilidad de producción escrita, como la variable de agrupación por género, al correlacionarlas, por tanto, con el promedio obtenido por desempeño (Hombre=2,8 y Mujer=3,3 con una D.E. 0,918 y 0,756, respectivamente), se obtiene un valor $\mathrm{p}=, 000^{\star * *}$ ( $\mathrm{p}=$ significancia de la prueba ANOVA), demostrando que la diferencia según género es significativa.

Por su parte, la Tabla 3, muestra que solamente el Colegio es un factor significativo dentro de los factores escolares para la habilidad de producción de textos con un valor de $\mathrm{p}=, 049^{*}$. No son factores significativos el curso o la especialidad, con valores de $p=, 343$ y $p=363$. 
Tabla 3.

Relación de Factores Escolares como Establecimiento, Curso y Especialidad con la Producción de un Ensayo Argumentativo.

\begin{tabular}{|c|c|c|c|c|}
\hline $\begin{array}{l}\text { Variable de } \\
\text { Agrupación }\end{array}$ & Valor & $\begin{array}{l}\text { Media de } \\
\text { Producción } \\
\text { Escrita }\end{array}$ & D.E. & p\# \\
\hline Establecimiento & $\begin{array}{l}\text { 1, de Administración Delegada } \\
\text { 2, de Administración Municipal }\end{array}$ & $\begin{array}{l}3,2 \\
3,0\end{array}$ & $\begin{array}{l}, 825 \\
, 837\end{array}$ &, $049^{*}$ \\
\hline Curso & $\begin{array}{l}3^{\circ} \text { Medio } \\
4^{\mathrm{a}} \text { Medio }\end{array}$ & $\begin{array}{l}3,0 \\
3,1\end{array}$ & $\begin{array}{l}, 820 \\
, 854\end{array}$ & ,343 \\
\hline Especialidad & $\begin{array}{l}\text { Administración } \\
\text { Contabilidad } \\
\text { Elaboración Industrial de } \\
\text { Alimentos } \\
\text { Secretariado Modalidad Dual } \\
\text { Servicio de Alimentación } \\
\text { Colectiva } \\
\text { Ventas }\end{array}$ & $\begin{array}{l}3,2 \\
3,2 \\
3,0 \\
3,2 \\
3,1 \\
3,0\end{array}$ & $\begin{array}{l}1,126 \\
, 690 \\
, 858 \\
\\
, 667 \\
, 823 \\
\\
, 788\end{array}$ & ,363 \\
\hline
\end{tabular}

\#: p se refiere a la significancia de la prueba ANOVA

En relación con la influencia de factores académicos en la producción de un ensayo, como las Notas de Enseñanza Media (NEM) y de la asignatura de Lenguaje y Comunicación (NLC) -tomadas estas últimas como variables de correlación-, al considerar que el Establecimiento 1 tiene un promedio NEM de 5,3 y Establecimiento 2 de 5,2, se obtiene un índice de correlación de 293 con un valor de $\mathrm{p}=, 000^{* * *}$ ( $\mathrm{p}$ se refiere a la significancia de la prueba Pearson), y el NLC de 5,2 en el Establecimiento 1 y 4,8, en el 2, se obtiene un índice de correlación de ,241 con un valor de $\mathrm{p}=, 001^{* *}$. En consecuencia, si bien ambas correlaciones son significativas, ellas son de bajo valor. Esto quiere decir que aún existen otros factores que pueden estar influyendo en su variabilidad.

\section{Discusión y conclusiones}

Este estudio indagó acerca del dominio de la competencia comunicativa escrita que presentan estudiantes $3^{\circ}$ y $4^{\circ}$ año de dos establecimientos de Educación Media Técnico Profesional (EMTP) de la Provincia de Nuble, mediante la evaluación de la producción de un ensayo argumentativo. Los resultados muestran que los alumnos de ambas unidades educativas alcanzan un 60,5\% de logro de la habilidad para escribir un texto argumentativo, lo que se traduce como un desempeño aceptable con 3,1 puntos. Lo anterior quiere decir que existe un manejo 
básico en gran parte de las dimensiones medidas, como los recursos del código ortográfico, de la coherencia y cohesión, de un léxico esencial y un claro propósito comunicativo que es el más logrado $(3,5)$, junto con el uso satisfactorio del espacio asignado (cobertura). Sin embargo, es limitado el empleo de las partes de la esquematización o división básica de un texto argumentativo, como se evidencia en los criterios Partes del Discurso (2,8) y en Argumentación (2,9). Los resultados negativos de estos dos últimos aspectos no concuerdan con lo que supuestamente debiera aplicar un estudiante que ya ha avanzado en su educación media, por cuanto, según los objetivos de aprendizaje del currículum nacional, en tercer año medio se enseña una unidad temática fundamental como es el discurso argumentativo.

Aunque desde las Bases Curriculares (2013) se establezca que la escritura es una de las competencias comunicativas claves en la formación de los estudiantes como mecanismo de transmisión y preservación del conocimiento, así como servir de vehículo para la expresión del sujeto y su desarrollo creativo, interactuar con los demás sin que el tiempo y espacio sean obstáculos, entre otras funciones, no es menos cierto que la habilidad de escribir es muy compleja, ya que involucra, según Álvarez (2010), conocimientos conceptuales, procedimentales y actitudinales de carácter social, cognitivo y retórico -principalmente-, y un gran desafío didáctico si quiere ser abordada. Existe evidencia de que en el aula escolar es escaso el fomento por la escritura argumentativa o crítica (Preiss, 2009, 2010), esto debido a que, como se mencionó anteriormente, los sistemas de medición como el SIMCE o la PSU hacen que los establecimientos prioricen el fortalecimiento de la habilidad de lectura, por sobre las otras, ya que es la más testeada. Solo recientemente (2013-2015) en $6^{\circ}$ Básico se evalúa la escritura de modo continuo y sistemático, donde se ha obtenido un puntaje promedio de 250 puntos SIMCE. Pero como todavía existe el rezago de su implementación en los niveles superiores, no se pueden comparar los resultados obtenidos de la muestra investigada. Por cierto, este proceso debe ir aparejado del diseño e implementación, tarea también pendiente, de los Estándares de Aprendizaje de Escritura, tal como existen para Lectura (MINEDUC, 2015).

Se puede hipotetizar que el Establecimiento 1 obtiene levemente un mejor resultado $(62,2 \%)$ que el Establecimiento $2(58,9 \%)$, debido a que imparte las especialidades de secretariado, administración y ventas, 
donde se puede estar incluyendo en la tarea formativa la redacción de variados textos funcionales que estos ámbitos requieren como cartas, formularios, solicitudes, guías, etc., tal como lo expresa, por ejemplo, el perfil genérico de Administración: "debe ser capaz de elaborar informes del desarrollo de un programa operativo de un departamento o área de una empresa [...] o aplicar técnicas de relaciones públicas, de comunicación oral y escrita, en forma presencial o a distancia" (p.16.), aunque existe una competencia genérica común para todos, que se orienta a: "Comunicarse oralmente y por escrito con claridad, utilizando registros de habla y escritura pertinentes a la situación laboral y a la relación con los interlocutores" (p.13). En cambio, en las especialidades de Elaboración Industrial de Alimentos y Servicio de Alimentación Colectiva del Establecimiento 2, su formación es más operativa (Cfr. MINEDUC, 2013b).

Si bien se ha constatado que en los dos últimos cursos de la Enseñanza Media no hay una evaluación de la escritura desde el sistema educacional, algunas instituciones sí realizan mediciones al ingreso de la Educación Superior. Es el caso de INACAP, a través del CEDEM (Centro de Desarrollo para la Educación Media) (2013), que en un diagnóstico aplicado a sus estudiantes de primer año en 2013 arrojó un promedio de 55,79\% de logro en producción de textos. Este es un dato relevante que se puede cotejar con el obtenido en esta investigación de 60,5\% de logro, ya que más del 60\% de los sujetos encuestados en esta muestra pretende seguir estudios en entidades como INACAP y similares. Esto, sin duda, demuestra que es necesario fortalecer la escritura escolar mediante programas o proyectos incipientes de alfabetización académica o de articulación con los desafíos discursivos de los estudios superiores desde el enfoque escribir para aprender (Navarro, 2013; Castelló, 2008; Marinkovich et al., 2009), apoyados por equipos especializados desde las universidades, preocupación que debe generar cada vez mejores condiciones para el aprendizaje, sobre todo, de alumnos vulnerables que requieren de ayudas concretas.

Por último, el desafío es continuar con nuevas investigaciones que permitan comprobar la capacidad predictiva de variables que se evidenciarán en el futuro, como es el puntaje PSU o las notas del primer 
año de Educación Superior, mediante un estudio longitudinal. Es posible también indagar acerca del desempeño en producción escrita en establecimientos de Educación Media Científico-Humanista y comparar los hallazgos obtenidos con la modalidad Técnico-Profesional.

\section{Referencias Bibliográficas}

Álvarez. T. (2010). Competencias básicas en escritura. Barcelona: Octaedro.

Castelló, M. (2008). Aprender a escribir, escribir para aprender. Aula de innovación educativa, 175, 7-9.

Chile. Agencia de Calidad de la Educación. (2015). Entrega de resultados de aprendizaje 2014Escritura. Recuperado de <http: / / archivos.agenciaeducacion.cl resultados2014/ Presentacion_Entrega_Resultados_2014_.pdf>

Chile. Centro de Desarrollo para la Educación Media (CEDEM). (2013). Reduciendo la brecha entre la Educación Media y la Educación Superior. Recuperado del sitio de Internet del Centro de Desarrollo para la Educación Media de INACAP: http://www.inacap.cl/web/2015/ sites / publicaciones/pdf/VinculoEducativoN2.pdf

Chile. Centro de Políticas y Prácticas de la Educación (CEPPE), Universidad Alberto Hurtado (UAH), Centro de Investigación Avanzada en Educación (CIAE). (2013). Formación de técnicos para Chile. ¿Un desafío sin políticas públicas? Recuperado del sitio de Internet del Centro de Investigación y Desarrollo de la Educación de la Universidad Alberto Hurtado: http: / / www.cide.cl/ documentos/Formacion_de_ tecnicos_para_Chile_CEPPE_UAH_CIAE.pdf

Chile. Ministerio de Educación (MINEDUC). (2010). Objetivos Fundamentales y Contenidos Minimos Obligatorios de la Enseñanza Básica y Media. Santiago: Unidad de Currículum y Evaluación. 
Chile. Ministerio de Educación (MINEDUC). (2013a). Bases Curriculares de Lengua y Literatura. Recuperado del sitio de Internet de Internet del Ministerio de Educación: http: / / www.curriculumenlineamineduc.cl / 605 / articles30013_recurso_17_08.pdf

Chile. Ministerio de Educación (MINEDUC). (2013b). Bases Curriculares. Formación Diferenciada Técnico-Profesional Especialidades y Perfiles de Egreso. Santiago. Recuperado del sitio de Internet del Ministerio de Educación: file:///D:/Downloads/Bases $\% 20$ formacion $\% 20$ TP\%20 (FINAL\%20nov\%202013)\%20 (1).pdf

Chile. Ministerio de Educación (MINEDUC). (2015). Estándares de Aprendizaje de Lectura Segundo Año Medio. Recuperado del sitio de Internet del Ministerio de Educación: / / www.curriculumenlineamineduc. cl/605/articles-33859_recurso_9.pdf

Chile. Departamento de Medición y Registro Académico (DEMRE). (2014). Intersección curricular decretos 220 y 254 para modelamiento de la PSU de Lenguaje 2015. Recuperado del sitio de Internet del Colegio Santa Teresita de Coelemu:http:/ / www.colegiosantateresitacoelemu. cl/documentos / Temarios_PSU/lyc_interseccion_ curricular_p2015.pdf

Chile. Laboratorio Latinoamericano de Evaluación de la Calidad de la Educación (LLECE). (2015). Tercer Estudio Regional Comparativo y Explicativo (TERCE). Recuperado del sitio de Internet de la UNESCO: http://www. unesco.org/new/es/education/

Cofré, V. et al. (2013). Lenguaje y comunicación $2^{\circ}$ Medio. Texto del estudiante. Santiago: SM.

Domínguez, C. et al. (2013). Lenguaje y comunicación $3^{\circ}$ Medio. Texto del estudiante. Santiago. SM.

Ducrot, O. (2001). El decir y lo dicho: polifonía de la enunciación. Barcelona: Paidós. 
Errázuriz, M. (2014). El desarrollo de la escritura argumentativa académica: los marcadores discursivos. Onomazein, 30, 217-236.

España. Real Academia Española (RAE). (2014). Diccionario de la lengua española. Recuperado de www.rae.es

Flotts, P. y Manzi, J. (2012). Medición de habilidades de comunicación escrita en estudiantes universitarios La experiencia UC. Recuperado del sitio de Internet del Centro MIDE UC de la Pontificia Universidad Católica de Chile: http: / / www.mideuc.cl/presentaciones_colmee/ Paulina_Flotts_Examen_Com_Escrita.pdf

Flower y Hayes. (1981). A cognitive process theory of writing. College Composition and Communication, 32, 365- 87.

Fox, J. (2005). The R Commander: A Basic Statistics Graphical User Interface to R. Journal of Statistical Software, 14(9), $1-42$.

Guin-Po, P. et al. (2001). Lengua Castellana y Comunicación $3^{\circ}$ Medio. Texto del estudiante. Santiago: SM.

Grabe, W.; Kaplan, R. (1996). Theory and Practice of Writing. Londres: Longman.

Hernández, R. et al. (2006). Metodología de la investigación. México: McGraw-Hill.

Hernández, J. (2009). La evaluación de la escritura en PSULenguaje y Comunicación: Estudios preliminares EXPERIMENTACIONES 2006-2007 DEMRE Recuperado del sitio de Internet del Centro MIDE UC de la Pontificia Universidad Católica de Chile: http: / / mideuc.cl/ wpcontent/ uploads / 2011/07/La evaluaciondelaescrituraenPSUleng.ppt>

Kellog, R. \& Whiteford, A. (2012). The development of writing expertise. En E. Grigorenko, E. Mambrino y D. Preiss (Eds). Writing: A mosaic of new perspectives. New York: Psychology Press. 
La Segunda. (2012). Anuncian profundos cambios a la PSU: Viene ensayo escrito e incluirán ranking del alumno. Recuperado del sitio de Internet del diario La Segunda: http: / / www. lasegunda.com/ Noticias/Nacional/2012/01/7102

Lo Cascio, V. (1998). Gramática de la argumentación. Madrid: Alianza.

Marinkovich, J. et al. (2009). Aprendiendo a escribir en las disciplinas. Articulación entre el currículum escolar y el universitario. Valparaíso: Ediciones Universitarias de Valparaíso.

Mertler, Craig A. (2001). Designing scoring rubrics for your classroom. Practical Assessment, Research \& Evaluation, 7(25). Recuperado de file:///C:/Documents $\% 20$ and $\% 20$ Settings / Part\% 20Time / Mis\%20documentos / Downloads/Metler_Designing_scoring_rubrics_ for_your_classroom\%20(1).pdf

Navarro, F. (2013). Escribir para aprender. Disciplinas y escritura en la escuela secundaria. Buenos: Paidós.

Neira, A.; Ferreira, A. (2011). Escritura académica: un modelo metodológico efectivo basado en tareas y enfoque cooperativo. Lingüistica y Literatura, 24, 143-159.

Parodi, G. (2003). Relaciones entre lectura y escritura: Una perspectiva cognitiva discursiva. Bases teóricas y antecedentes empíricos. Valparaíso: Ediciones Universitarias de Valparaíso.

Preiss, D. (2009). The Chilean instructional pattern for the teaching of language: A video-survey study based on a national program for the assessment of teaching. Learning and Individual Differences, 19, 1-11.

Preiss, D. (2010). Folk pedagogy and cultural markers in teaching: Three illustrations from Chile. In D. D. Preiss, \& R. J. Sternberg (Eds.), Innovations in educational psychology: Perspectives on teaching, learning and human development (pp. 325-355). New York: Springer Publishing Company. 
Preiss, D. et al. (2013). Assessment of argumentative writing and critical thinking in higher education: Educational correlates and gender differences, Learning and Individual Differences, 28, 193-203.

R Core Team. (2013). R: A language and environment for statistical computing. $R$ Foundation for Statistical Computing, Vienna, Austria. Recuperado de http:/ / www.R-project.org/

Van Dijk, T. (1997). La ciencia del texto. Barcelona: Paidós.

Weinberg, L. (2006). Pensar el ensayo. México: Fondo Cultura Económica.

Weinstein, A. (2013). Diagnóstico de la Educación Media Técnico-Profesional: Estado de Situación y Agenda Futura. Recuperado del sitio de Internet de Libertad y Desarrollo: http:// www.lyd.org/wp-content/themes / LYD/files_mf/ siso143 